Article

\title{
Time Dependence of Ultra-Short Laser Pulses Scattering by Atom in High Frequency Limit
}

\author{
Valeriy Alexandrovich Astapenko ${ }^{1, *}$, Frank Bernhard Rosmej ${ }^{1,2,3}$ and \\ Egor Sergeevich Khramov ${ }^{1}$ \\ 1 Moscow Institute of Physics and Technology, National Research University, Dolgoprudny 141701, Russia; \\ frank.rosmej@sorbonne-universite.fr (F.B.R.); egor.khramov@phystech.edu (E.S.K.) \\ 2 Faculty of Science and Engineering, Sorbonne University, 75006 Paris, France \\ 3 LULI, Ecole Polytechnique, CEA, CNRS, Laboratoire pour l'Utilisation des Lasers Intenses, \\ F-91128 Palaiseau, France \\ * Correspondence: astapenko.va@mipt.ru
}

Received: 17 July 2020; Accepted: 6 August 2020; Published: 7 August 2020

check for updates

\begin{abstract}
We investigated theoretically the time dependence of ultra-short laser pulse scattering by an atom at the high-frequency limit for the spectral and total probability of the process using new expression which we derived in this paper. We established that the time dependence of spectral scattering is presented by the curve with the maximum for sufficiently large detuning of scattering frequency from the carrier frequency of the pulse, while the total scattering probability is always the monotonically increasing function of time. We also studied the dependence of scattering probability on pulse duration at the long-time limit. It was shown that, at the long-pulse limit, the scattering probability is a linear function of pulse duration, while in the opposite case, it is a function with maximum. The position of this maximum is determined by the detuning of the scattering frequency from the carrier frequency of the pulse.
\end{abstract}

Keywords: ultra-short laser pulse; radiation scattering; time dependence; spectral scattering probability

\section{Introduction}

Ultra-short laser pulses' (USLP) interactions with matter are one of the hottest topics in modern science that is proven by the variety of possible applications [1-5].

Due to the development of the technique for generating USLP and the methods for observing photo processes in real time on the femto- and subfemtosecond time scale [6-14], it is necessary to adequately describe the time dependence of the probability of absorption and the scattering of radiation by atoms.

At the present time, multiple methods for the description of atomic processes in the high-frequency field exist (e.g., see [15]). However, the field of USLP is very different. As was shown in a number of previous works [16-22], the standard approach to the description of photo processes in the ultra-short pulse field, based on probabilities per unit time, is not always adequate, since it does not take into account the finite width of the ultra-short pulse spectrum. In some articles [21,22], it was proposed to use the probability for the entire duration of the USLP as the main characteristic of the radiative process in the field of USLP. In this way, the scattering of ultra-short pulses by various targets was studied-both Rayleigh, including the resonance case, and Compton [18-20]. The most attention was paid to the dependence of the probability on the pulse duration. In particular, criteria were established for the nonlinear nature of this dependence. Note that when using the probability per unit time, this dependence is always linear. 
The scattering spectra of ultra-short pulses on atomic and molecular targets in the case in which the pulse duration is much shorter than the characteristic atomic time were calculated in some papers [23-26] using the sudden perturbation method. In these works, the dependence of the probability of the photo process upon the pulse duration were not studied, and in addition, the condition for the applicability of the approximation of sudden perturbations imposes very strong restrictions on the shortness of the pulse.

Besides the probability of the photo process for the entire duration of the USLP, it is of interest to investigate the dependence of the probability on time. Thus, the time dependence of the ionization of a hydrogen atom by ultra-short pulses has been studied [27] using a numerical solution of the temporal Schrödinger equation. Particularly, it was shown that this dependence is strongly non-monotonic with maxima and minima.

The attosecond dynamics of the excitation of a hydrogen atom in a discrete and continuous spectrum was considered in one paper [28] in the framework of perturbation theory. In a previous article [29], we investigated the temporal evolution of the resonant scattering of ultra-short pulses by an atom and derived the general expression for the probability of this process. In particular, it was shown that, depending on the pulse duration and detuning of carrier frequency from its own atomic frequency, the spectral probability of resonant scattering can be an oscillatory function of time.

The present paper is devoted to the investigation of another limiting case in the USLP scattering by atom, namely the scattering of ultra-short pulses with a carrier frequency much higher than the characteristic atomic frequencies.

\section{General Formulas}

The general formula describing the time dependence of USLP spectral scattering probability by an atom in dipole approximation has the following form [30] (we use atomic units throughout the paper: $e=m=\hbar=1$ ):

$$
\frac{d W(t)}{d \omega^{\prime}}=\frac{\omega^{\prime 3}}{6 \pi^{3} c^{3}}\left|\int_{-\infty}^{t} d t^{\prime} \exp \left(i \omega^{\prime} t^{\prime}\right) \int_{-\infty}^{\infty} c_{11}\left(\omega^{\prime}, \widetilde{\omega}\right) E(\widetilde{\omega}, \omega, \tau) \exp \left(-i \widetilde{\omega} t^{\prime}\right) d \widetilde{\omega}\right|^{2}
$$

Here, $\omega^{\prime}$ is the frequency of the scattered radiation, $\omega$ is the carrier frequency, $\tau$ is the pulse duration, and $c$ is the velocity of light. $E(\widetilde{\omega}, \omega, \tau)$ is the Fourier transform of the electric field strength with amplitude $E_{0}$ in incident USLP [19]:

$$
E(\widetilde{\omega}, \omega, \tau)=i E_{0} \tau \sqrt{\frac{\pi}{2}} \frac{\widetilde{\omega}^{2} \tau^{2}}{1+\omega^{2} \tau^{2}}\left[\exp \left(-\frac{(\widetilde{\omega}-\omega)^{2} \tau^{2}}{2}\right)-\exp \left(-\frac{(\widetilde{\omega}+\omega)^{2} \tau^{2}}{2}\right)\right]
$$

$c_{11}\left(\omega^{\prime}, \widetilde{\omega}\right)$ is the scattering tensor of the radiation which for the atom reduces to the scalar:

$$
c_{11}\left(\omega^{\prime}, \widetilde{\omega}\right)=\sum_{n}\left|d_{n 1}\right|^{2}\left\{\frac{1}{\omega_{n 1}-\widetilde{\omega}-i \gamma_{n 1}}+\frac{1}{\omega_{n 1}+\omega^{\prime}-i \gamma_{n 1}}\right\}
$$

where $\omega_{n 1}$ and $\gamma_{n 1}$ are the eigenfrequencies and the relaxation constants of electron transitions, and $d_{n 1}$ are the matrix elements of the electric dipole moment.

In the high-frequency limit, $\omega>>I_{a}\left(I_{a}\right.$ is the atomic ionization potential) and the dipole approximation Equation (3) simplifies to the following form [28]:

$$
c_{11}\left(\omega^{\prime}, \widetilde{\omega}\right) \approx-\frac{N_{a}}{\omega^{\prime} \widetilde{\omega}}
$$


Here, $N_{a}$ is the number of atomic electrons. Substituting (4) in (1), we have

$$
\frac{d W(t)}{d \omega^{\prime}}=\frac{\omega^{\prime} N_{a}^{2}}{6 \pi^{3} c^{3}}\left|\int_{-\infty}^{t} d t^{\prime} \exp \left(i \omega^{\prime} t^{\prime}\right) \int_{-\infty}^{\infty} \frac{E(\widetilde{\omega}, \omega, \tau)}{\widetilde{\omega}} \exp \left(-i \widetilde{\omega} t^{\prime}\right) d \widetilde{\omega}\right|^{2} .
$$

Note that

$$
\int_{-\infty}^{\infty} \frac{E(\widetilde{\omega}, \omega, \tau)}{\widetilde{\omega}} \exp (-i \widetilde{\omega} t) d \widetilde{\omega}=-2 \pi i \int_{-\infty}^{t} E\left(t^{\prime}, \omega, \tau\right) d t^{\prime}
$$

Using Equation (6), we obtain from (5):

$$
\frac{d W(t)}{d \omega^{\prime}}=\frac{2 \omega^{\prime} N_{a}^{2}}{3 \pi c^{3}}\left|\int_{-\infty}^{t} d t^{\prime} \exp \left(i \omega^{\prime} t^{\prime}\right) \int_{-\infty}^{t^{\prime}} E\left(t^{\prime \prime}, \omega, \tau\right) d t^{\prime \prime}\right|^{2}
$$

\section{Results and Discussion}

Next, we calculate the time and spectral dependences of the scattered probability of the USLP by the atom in the high-frequency limit using Equation (7). All calculations are made for $\mathrm{Na}=1$ and for the amplitude of electric field in the pulse $\mathrm{E} 0=1$ a.u.

The time evolution of the scattering probability spectrum is presented in Figure 1 for the carrier frequency $\omega=20$ a.u. and pulse duration $\tau=50$ a.u.

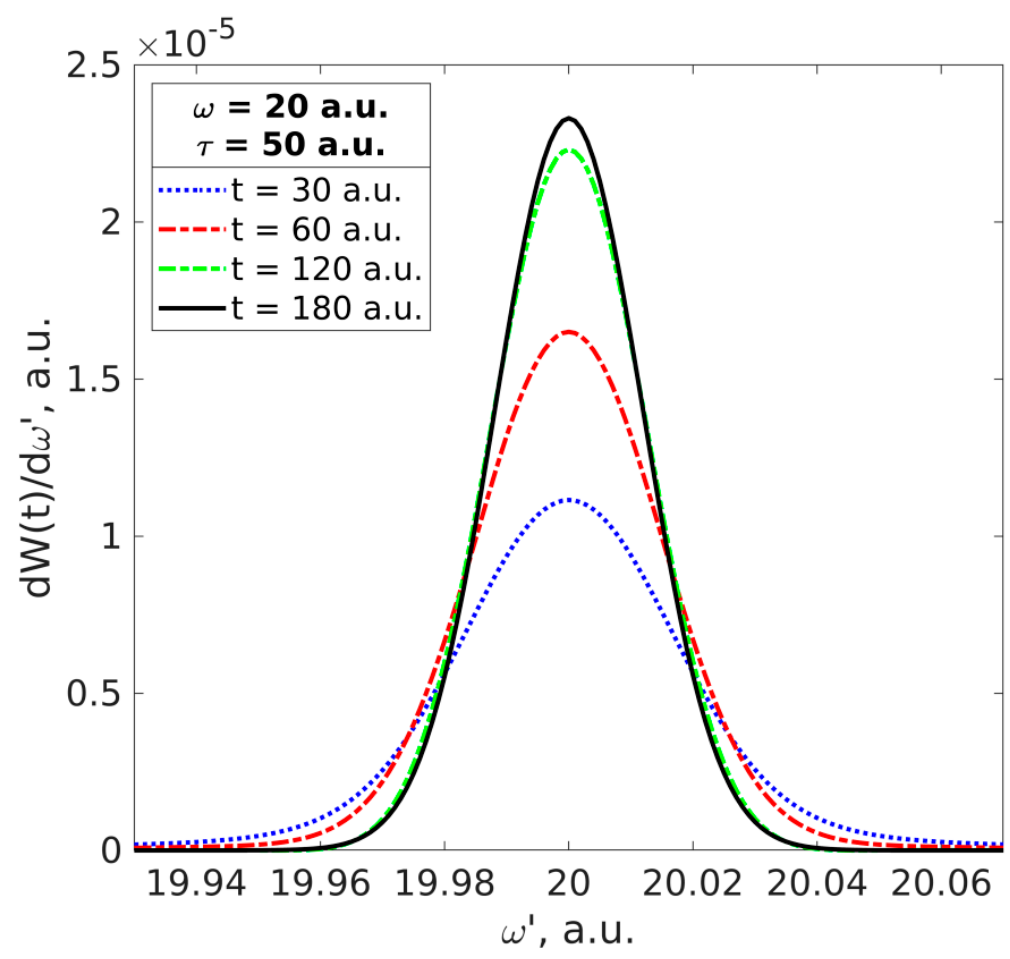

Figure 1. Scattering probability spectrum at different points in time.

One can see that the scattering spectrum has a bell-like shape and the spectral width decreases with the increase in time. In the long-time limit, the spectral width is determined by the reciprocal value of pulse duration (see Equation (10) below).

The results of the calculation of the time dependence of the spectral scattering probability in the high-frequency limit are shown in Figure 2 for various pulse durations and the scattering frequency. 

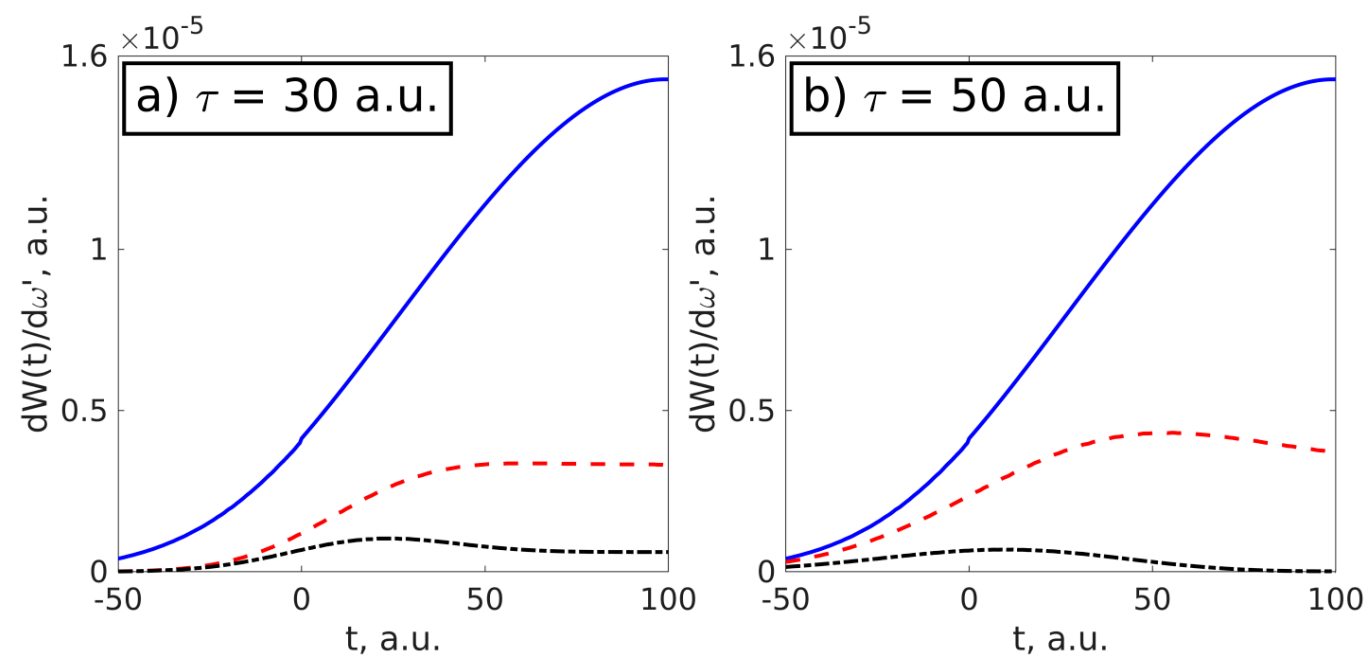

Figure 2. Time dependence of spectral scattering probability at different frequencies of scattered radiation. Solid line $-\omega^{\prime}=20$ a.u., dashed line $-\omega^{\prime}=20.025$ a.u., dash-dot line $-\omega^{\prime}=20.05$ a.u. Carrier frequency- $\omega=20$ a.u.

As can be seen from Figure 2, the time dependence of the spectral scattering probability is determined by nonadiabaticity parameter $\Delta=\left|\omega^{\prime}-\omega\right| \tau$. For $\Delta<1$, the spectral scattering probability is the monotonically increasing function of time; in the opposite case, it has a maximum.

For total scattering probability as a function of time, it follows from (7) and can be expressed as the following:

$$
W(t)=\int_{0}^{\infty} \frac{2 \omega^{\prime} N_{a}^{2}}{3 \pi c^{3}}\left|\int_{-\infty}^{t} d t^{\prime} \exp \left(i \omega^{\prime} t^{\prime}\right) \int_{-\infty}^{t^{\prime}} E\left(t^{\prime \prime}, \omega, \tau\right) d t^{\prime \prime}\right|^{2} d \omega^{\prime} .
$$

It should be mentioned that the main contribution to the integral (8) arises from the frequency range in the vicinity of the carrier frequency of the pulse which satisfied the inequality $\omega>>I_{a}$. Consequently, the high frequency approximation for the scattering tensor (4) is valid.

The time dependence of the total scattering probability is shown in Figure 3 for different pulse durations.

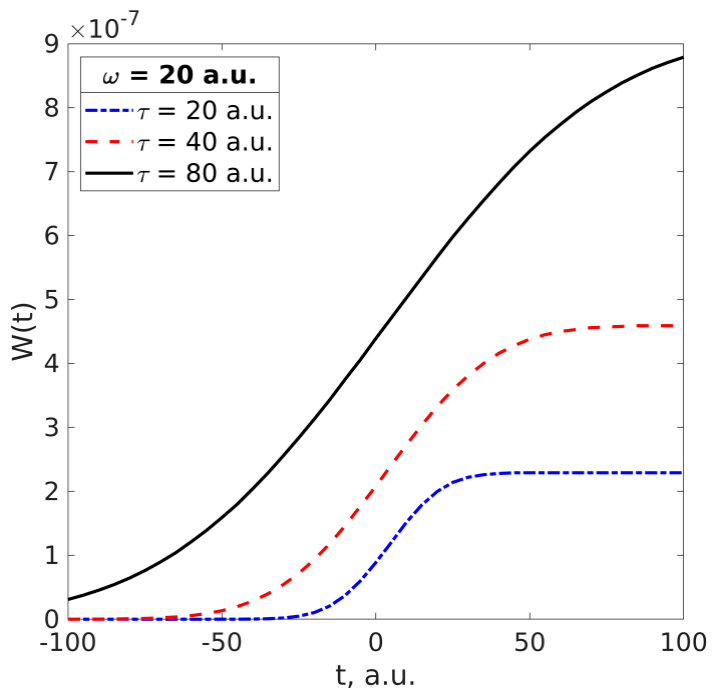

Figure 3. The total scattering probability as the function of time for various pulse durations.

As can be seen from this figure, the total probability is always a monotonically increasing function of time, in contrast to the spectral probability of the USLP scattering. 
In the long-time limit $t \rightarrow \infty$, Equation (5) gives

$$
\frac{d W(\tau)}{d \omega^{\prime}}=\frac{2 N_{a}^{2}}{3 \pi \omega^{\prime} c^{3}}\left|E\left(\omega^{\prime}, \omega, \tau\right)\right|^{2} .
$$

Note that Equation (9) coincides, up to a non-dipole term, with expression (23), obtained in [16] in the framework of the approximation of sudden perturbations for the total spectrum of the scattering of ultra-short pulses by a hydrogen atom $\left(N_{a}=1\right)$.

For the scattering of laser pulse with Gaussian envelope, we have from (9) the following:

$$
\frac{d W(\tau)}{d \omega^{\prime}}=\frac{N_{a}^{2}}{3 \omega^{\prime} c^{3}} E_{0}^{2} \tau^{2} \exp \left[-\left(\omega-\omega^{\prime}\right)^{2} \tau^{2}\right] .
$$

In the long pulse limit, Equation (10) gives

$$
\frac{d W(\tau \rightarrow \infty)}{d \omega^{\prime}}=\frac{\sqrt{\pi} N_{a}^{2}}{3 \omega^{\prime} c^{3}} E_{0}^{2} \tau \delta\left(\omega-\omega^{\prime}\right) .
$$

Thus, in this case, the scattering probability is the linear function of the pulse duration, in accordance with the standard approach, and the scattering frequency coincides with the carrier frequency because of the delta function in (11): $\omega^{\prime}=\omega$.

It is easy to calculate the pulse duration value at which the probability (10) has a maximum:

$$
\tau_{\max }=\frac{1}{\left|\omega-\omega^{\prime}\right|}, \omega^{\prime} \neq \omega .
$$

In the case when $\omega^{\prime}=\omega$, we have the following:

$$
d W(\tau) / d \omega^{\prime} \propto \tau^{2} .
$$

The validity of Equations (12) and (13) is demonstrated by curves in Figure 4 presenting the dependence of spectral scattering probabilities as functions of pulse duration for different scattering frequencies.

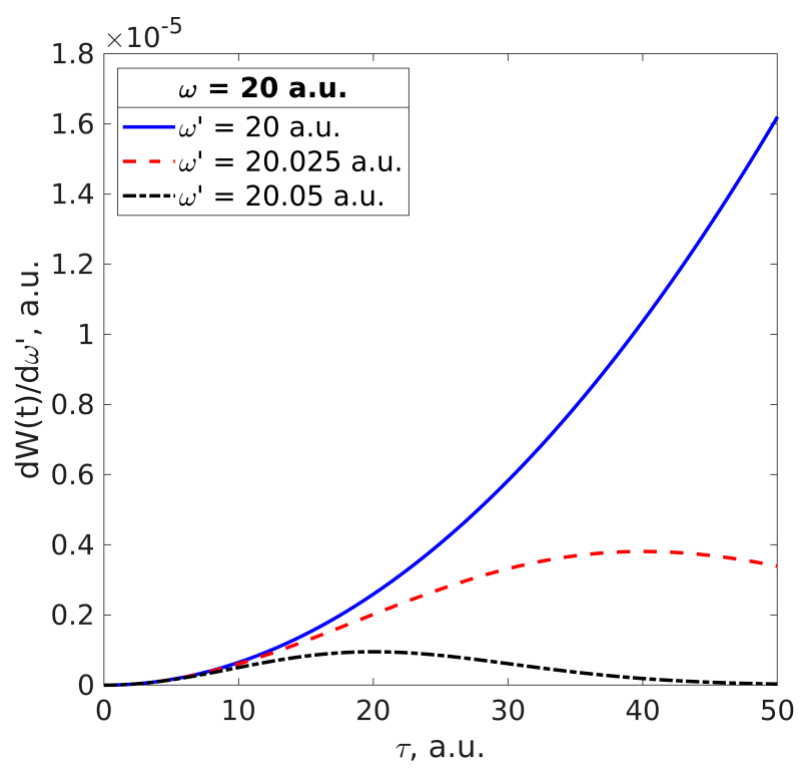

Figure 4. The spectral scattering probability dependence on the pulse duration in the long-time limit for different scattering frequencies. 
In reality, in the case $\omega^{\prime}=\omega$, the scattering probability increases quadratically with the pulse duration, and the position of maximum for $\omega^{\prime} \neq \omega$ satisfies Equation (12).

\section{Conclusions}

In this paper, we studied the scattering of USLP by the atom for carrier frequencies of the pulse which are much higher than characteristic atomic frequencies. Calculations were made for the scattering probability as the function of time and pulse duration in the dipole approximation and the second order of the perturbation theory. We derived a simple expression for the spectral scattering probability time dependence through the double integral of the electric field strength in the pulse (7).

It was shown that the spectral scattering probability transforms from the monotonically increasing function of time into function with maximum with the increase in the nonadiabacity parameter $\Delta=\left|\omega^{\prime}-\omega\right| \tau$, while the total scattering probability is always monotonically increasing with time.

We also demonstrated that, in the long-time limit, the dependence upon time disappears and the spectral scattering probability is proportional to the squared modulus of the Fourier transform of the electric field strength at the scattering frequency. In this case, the function $d W(\tau) / d \omega^{\prime}$ transforms from a quadratically to linearly increasing function and has a maximum at $\tau_{\max }=1 /\left|\omega^{\prime}-\omega\right|$ for $\omega^{\prime} \neq \omega$. In the long-pulse limit, the scattering spectrum shifts to the delta function with $\omega^{\prime}=\omega$ and the scattering probability is a linear function of the pulse duration.

Author Contributions: All authors made equal contribution to the paper. All authors have read and agreed to the published version of the manuscript.

Funding: This research was funded by RFBR, Project No. 19-32-90016.

Acknowledgments: This research was supported by the Moscow Institute of Physics and Technology in the framework of the 5-Top-100 program (project no. 075-02-2019-967).

Conflicts of Interest: The authors declare no conflict of interest.

\section{References}

1. Lawrence, J.R.; Waugh, D. Laser Surface Engineering: Processes and Applications; Elsevier: Amsterdam, The Netherlands, 2014.

2. Shiner, A.; Schmidt, B.; Trallero-Herrero, C.; Wörner, H.J.; Patchkovskii, S.; Corkum, P.B.; Kieffer, J.; Légaré, F.; Villeneuve, D. Probing collective multi-electron dynamics in xenon with high-harmonic spectroscopy. Nat. Phys. 2011, 7, 464. [CrossRef]

3. Yakovlev, V.S.; Gagnon, J.; Karpowicz, N.; Krausz, F. Attosecond streaking enables the measurement of quantum phase. Phys. Rev. Lett. 2010, 105, 073001. [CrossRef] [PubMed]

4. Lindroth, E.; Calegari, F.; Young, L.; Harmand, M.; Dudovich, N.; Berrah, N.; Smirnova, O. Challenges and opportunities in attosecond and XFEL science. Nat. Rev. Phys. 2019, 1, 107. [CrossRef]

5. Athanasiou, C.E.; Hongler, M.O.; Bellouard, Y. Unraveling brittle-fracture statistics from intermittent patterns formed during femtosecond laser exposure. Phys. Rev. Appl. 2017, 8, 054013. [CrossRef]

6. Tanaka, T. Proposal to generate an isolated monocycle $\mathrm{x}$-ray pulse by counteracting the slippage effect in free-electron lasers. Phys. Rev. Lett. 2015, 114, 044801. [CrossRef]

7. Kida, Y.; Kinjo, R.; Tanaka, T. Synthesizing high-order harmonics to generate a sub-cycle pulse in free-electron lasers. Appl. Phys. Lett. 2016, 109, 151107. [CrossRef]

8. Chini, M.; Zhao, K.; Chang, Z. The generation, characterization and applications of broadband isolated attosecond pulses. Nat. Photon. 2014, 8, 178-186. [CrossRef]

9. Mitrofanov, A.V.; Sidorov-Biryukov, D.A.; Glek, P.B.; Rozhko, M.V.; Stepanov, E.A.; Shutov, A.D.; Ryabchuk, S.V.; Voronin, A.A.; Fedotov, A.B.; Zheltikov, A.M. Chirp-controlled high-harmonic and attosecond-pulse generation via coherent-wake plasma emission driven by mid-infrared laser pulses. Opt. Lett. 2020, 45, 750-753. [CrossRef]

10. Beaulieu, S.; Comby, A.; Clergerie, A.; Caillat, J.; Descamps, D.; Dudovich, N.; Fabre, B.; R Géneaux, R.; Légaré, F.; Petit, S.; et al. Attosecond-resolved photoionization of chiral molecules. Science 2017, 358, 1288-1294. [CrossRef] 
11. Isinger, M.; Squibb, R.J.; Busto, D.; Zhong, S.; Harth, A.; Kroon, D.; Nandi, S.; Arnold, C.L.; Miranda, M.; Dahlström, J.M.; et al. Photoionization in the time and frequency domain. Science 2017, 358, 893-896. [CrossRef]

12. Hütten, K.; Mittermair, M.; Stock, S.; Beerwerth, R.; Shirvanyan, V.; Riemensberger, J.; Duensing, A.; Heider, R.; Wagner, M.S.; Guggenmos, A.; et al. Ultrafast quantum control of ionization dynamics. EPJ Web Conf. 2019, 205, 06001. [CrossRef]

13. Kaldun, A.; Blättermann, A.; Donsa, S.; Stooß, V.; Wei, H.; Pazourek, R.; Nagele, S.; Ott, C.; Lin, C.D.; Burgdörfer, J.; et al. Observing the ultrafast buildup of a Fano resonance in the time domain. Science 2016, 354, 738-740. [CrossRef] [PubMed]

14. Gruson, V.; Barreau, L.; Jiménez-Galan, Á.; Risoud, F.; Caillat, J.; Maquet, A.; Carré, B.; Lepetit, F.; Hergott, J.-F.; Ruchon, T.; et al. Attosecond dynamics through a Fano resonance: Monitoring the birth of a photoelectron. Science 2016, 354, 734-737. [CrossRef] [PubMed]

15. Oks, E. Analytical Advances in Quantum and Celestial Mechanics: Separating Rapid and Slow Subsystems; IOP Publishing Ltd.: Bristol, UK, 2020.

16. Rosmej, F.B.; Astapenko, V.A.; Lisitsa, V.S. Generalized scaling laws for ionization of atomic states by ultra-short electromagnetic pulses. J. Phys. B 2016, 49, 025602. [CrossRef]

17. Astapenko, V.A.; Lisitsa, V.S. Interaction of ultrashort laser pulses with atoms in plasmas. Atoms 2018, 6, 38. [CrossRef]

18. Rosmej, F.B.; Astapenko, V.A.; Lisitsa, V.S. XUV and x-ray elastic scattering of attosecond electromagnetic pulses on atoms. J. Phys. B 2017, 50, 235601. [CrossRef]

19. Astapenko, V.A.; Moroz, N.N.; Mutafyan, M.I. Compton scattering of attosecond x-ray pulses on a hydrogen atom. JETP Lett. 2018, 108, 165-169. [CrossRef]

20. Rosmej, F.B.; Astapenko, V.A.; Lisitsa, V.S.; Moroz, N.N. Nonlinear resonance scattering of femtosecond X-ray pulses on atoms in plasmas. Phys. Lett. A 2017, 381, 3576-3579. [CrossRef]

21. Astapenko, V.A. Simple formula for photoprocesses in ultrashort electromagnetic field. Phys. Lett. A 2010, 374, 1585-1590. [CrossRef]

22. Astapenko, V.A. Scattering of ultrashort electromagnetic radiation pulse by an atom in a broad spectral range. JETP 2011, 112, 193-198. [CrossRef]

23. Matveev, V.I. Emission and electron transitions in an atom interacting with an ultrashort electromagnetic pulse. JETP 2003, 97, 915-921. [CrossRef]

24. Makarov, D.N.; Matveev, V.I. Spectra for the reemission of attosecond and shorter electromagnetic pulses by multielectron atoms. JETP 2017, 125, 189-194. [CrossRef]

25. Goshev, A.A.; Eseev, M.K.; Makarov, D.N. Scattering of attosecond electromagnetic field pulses by molecular anions including the magnetic field component. JETP 2020, 130, 28-34. [CrossRef]

26. Makarov, D.N. Quantum theory of scattering of ultrashort electromagnetic field pulses by polyatomic structures. Opt. Express 2019, 27, 31989-32008. [CrossRef]

27. Prasad, V.; Dahiya, B.; Yamashita, K. Ionization of the $\mathrm{H}$ atom in ultrashort chirped laser pulses. Phys. Scr. 2010, 82, 055302. [CrossRef]

28. Astapenko, V.A. Attosecond dynamics of photoexcitation of the hydrogen atom by ultrashort laser pulses. JETP 2020, 130, 56-61. [CrossRef]

29. Astapenko, V.A. Temporal dynamics of resonant scattering of an ultrashort laser pulse by an atom. Appl. Phys. $B$ 2020, 126, 110. [CrossRef]

30. Rosmej, F.B.; Astapenko, V.A.; Lisitsa, V.S. Plasma Atomic Physics; Springer Series on Atomic, Optical and Plasma Physics 104; Springer: Berlin/Heidelberg, Germany, 2020.

(C) 2020 by the authors. Licensee MDPI, Basel, Switzerland. This article is an open access article distributed under the terms and conditions of the Creative Commons Attribution (CC BY) license (http://creativecommons.org/licenses/by/4.0/). 